\title{
Kinematics and backgrounds for HAPPEX measurements
}

\author{
B. Moffit ${ }^{\mathrm{a}}$ \\ The College of William \& Mary, Department of Physics, Williamsburg, VA 23187-8795, USA
}

Received: 31 August 2006

Published online: 3 July 2007 - (C) Società Italiana di Fisica / Springer-Verlag 2007

\begin{abstract}
Measurements and systematic errors of the 4-momentum transfer squared $\left(Q^{2}\right)$ and backgrounds for the 2005 HAPPEX runs are described.
\end{abstract}

PACS. 12.15.-y Electroweak interactions - 25.30.Bf Elastic electron scattering - 07.05.Kf Data analysis: algorithms and implementation; data management

\section{Introduction}

Recent HAPPEX measurements have the goal of determining parity-violating asymmetries with very high absolute and relative precision in order to provide tight constraints on the strange electric and magnetic form factors of the proton. Accurate knowledge of the systematic errors that arise from experimental kinematics and contribution from backgrounds are needed in order to ensure that these measurements are statistically limited. The High Resolution Spectrometers (HRS) of Hall A at Jefferson Lab are the key feature in the achievement of this goal. In this paper we present the technique used for the measurement of the 4-momentum transfer squared $\left(Q^{2}\right)$ between the longitudinally polarized electron and unpolarized target nucleus, with particular focus on optics optimization and the target-recoil technique used to determine the central scattering angle. A detailed view of the determination and magnitude of backgrounds that contribute to the experimental asymmetry will also be presented.

\section{Kinematics determination}

The 4-momentum transfer squared $\left(Q^{2}\right)$ sets the kinematic scale of the asymmetry measurement, and is defined as

$$
Q^{2}=2 E E^{\prime}(1-\cos \theta),
$$

where $E$ is the beam energy, and $E^{\prime}$ and $\theta$ are the energy and angle of the scattered electron, respectively.

The beam energy is measured using dispersion in the $34.3^{\circ}$ bend which brings the beam into the experimental hall, detailed in ref. [1]. The parameters of the scattered electron are determined by reconstructing its path from the target to the spectrometer focal plane.

\footnotetext{
a Presenting on behalf of the HAPPEX Collaboration; e-mail: moffit@jlab.org
}
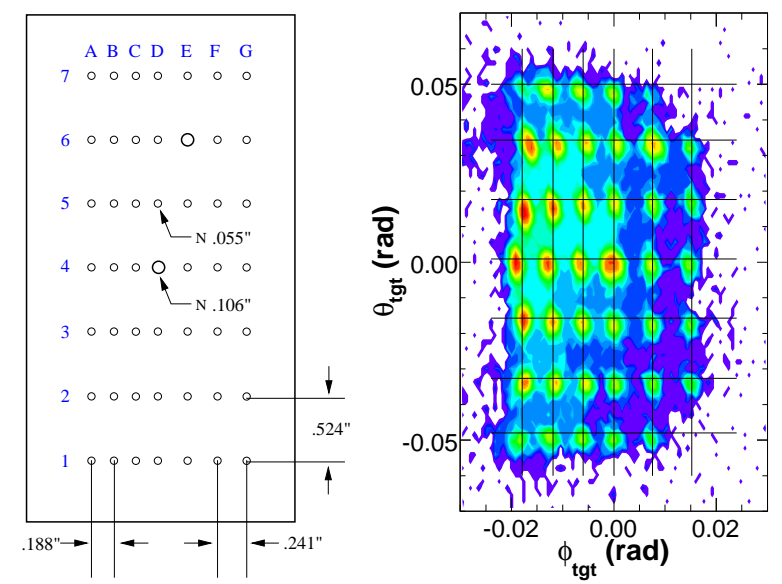

Fig. 1. Insertable sieve slit (left) and reconstructed sieve hole pattern (right).

\subsection{Optics optimization}

Scattered electrons are detected in the HRS using two planes of vertical-drift chambers to measure their position and angle in the focal plane. Combined with incident beam information, the track parameters at the target are reconstructed using transfer matrices optimized for the spectrometer optics. Optics optimization is aided by the use of a sieve slit: a $5 \mathrm{~mm}$ thick collimator placed in front of the acceptance of the first magnetic element of the spectrometer. The sieve slit (shown in fig. 1) contains a pattern of 49 pin holes $(7 \times 7)$ with two larger holes to allow for unambiguous identification of the orientation of the image at the focal plane. The known momentum, position, and angle of tracks from elastic scattering from a thin target through individual sieve-holes is used to optimize a polynomial expansion relating the trajectory in the focal plane 


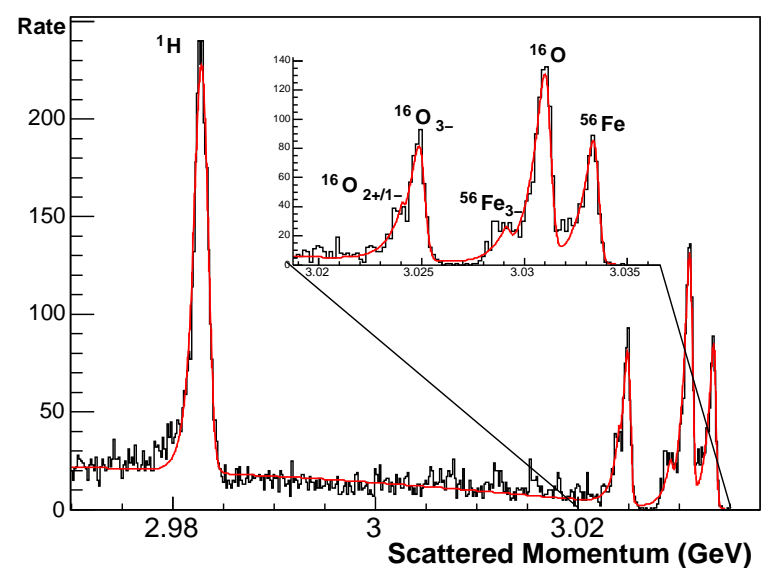

Fig. 2. Scattered momentum spectrum observed from the water cell through a single sieve hole. The solid-line fit to the data includes information about the mass of each state as well as radiative energy corrections.

to parameters at the target, as outlined in ref. [2]. Optics targets, located at $Z_{\text {lab }}=-12 \mathrm{~cm}, 0 \mathrm{~cm},+12 \mathrm{~cm}$, allow for optimization of the reconstruction along the full axis of the target.

Systematic errors from reconstruction are evaluated by applying ad hoc corrections to the observed sieve-slit pattern (also in fig. 1) to remove the small deviations from expected locations. The observed shift in $Q^{2}$ is found to be small $(\leq 0.3 \%)$ and is not corrected for.

\subsection{Central angle measurement}

The absolute calibration of the spectrometer central scattering angle was made with calibration data from a water cell target. This target was a $0.5 \mathrm{~mm}$ thick container of flowing water, with 1 mil steel windows. From relativistic kinematics, the scattered electron energy $E^{\prime}$ can be found in terms of the scattering angle $\theta$ :

$$
E^{\prime}=\frac{E-\frac{1}{2 m}\left(m^{* 2}-m^{2}\right)}{1+\frac{E}{m}(1-\cos \theta)},
$$

where $E$ is the beam energy, $m$ is the mass of the target nucleus, and $m^{*}$ is the mass of the recoil state. Figure 2 shows a fit to the resulting measured energy spectrum from this target. The momentum separation between the hydrogen and heavier recoil peaks was used to determine the central angle with an uncertainty of $0.2 \%$.

\subsection{Corrections}

In production mode, the signals from the HAPPEX detector are integrated over a $33 \mathrm{~ms}$ gate. This means that the asymmetry measurement is implicitly weighted by the energy deposited by the incoming electron. The $Q^{2}$ measurement is performed in tracking mode, therefore each
Table 1. Summary of the systematic errors in $Q^{2}$.

\begin{tabular}{lc}
\hline Source & Percent Error in $Q^{2}$ \\
\hline Beam Energy & $0.1 \%$ \\
HRS Momentum Scale & $0.2 \%$ \\
Optics Optimization & \multicolumn{2}{c}{$0.3 \%$} \\
At $Z=0$ & $0.1 \%$ \\
$Z$ dependence & $0.4 \%$ \\
Scattering Angle & He: $0.1 \% \quad$ H: $0.5 \%$ \\
ADC Weighting & $0.6 \%$ \\
Drifts in time & He: $0.3 \% \quad$ H: $0.1 \%$ \\
Rate Dependence & He: $0.9 \% \quad$ H: $1.0 \%$ \\
\hline Total Error &
\end{tabular}

track hitting the detector must be explicitly weighted by its ADC response. An upper limit to the systematic of this weighting was taken to be the full size of the corrections: $0.1 \%$ for the helium run, and $0.5 \%$ for the hydrogen run.

A drift over time was observed for these measurements in the helium run period, with the full scale of the drift taken to be an upper limit on the uncertainty of $0.6 \%$. Although no such drift was apparent in the hydrogen run period, this error estimate was included in both data sets.

A series of measurements was taken at various beam currents to check for possible systematic errors due to ratedependent effects in reconstruction, resulting in an additional estimated uncertainty of $0.3 \%$ for helium and $0.1 \%$ for the hydrogen runs.

Table 1 presents a summary of the systematic errors in $Q^{2}$.

\section{Backgrounds}

There are three main contributors to the backgrounds in HAPPEX: scattering from the target aluminum walls, quasielastic scattering from ${ }^{4} \mathrm{He}$, and rescattering in the spectrometer.

\subsection{Target walls}

Background from scattering from the target aluminum walls was analyzed using a pair of aluminum foils positioned at $Z_{\text {lab }}= \pm 10 \mathrm{~cm}$ (the location of the target cell's endcaps). The thickness of the foils $(1.0 \mathrm{~mm})$ was chosen to match the radiation length of the full target cell. The background fraction $\left(f_{\mathrm{Al}}\right)$ is then determined from

$$
f_{\mathrm{Al}}=x_{t} \frac{R_{\mathrm{Al} \mathrm{Foils}}}{R_{\text {Target Cell }}},
$$

where $x_{t}$ is the ratio of the target cell wall thickness to the dummy cell wall thickness, and $R_{\mathrm{Al}}$ Foils(Target Cell) is the normalized scattering rate from the aluminum foils (target cell). With this method, the aluminum fraction for helium was $(1.8 \pm 0.2) \%$ and for hydrogen was $(0.7 \pm 0.3) \%$. The uncertainty is dominated by the uncertainty in the 


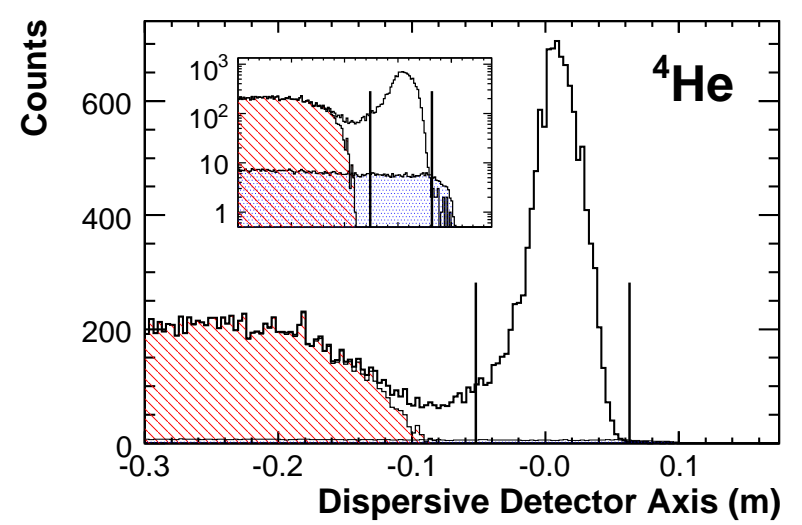

Fig. 3. Spectra of scattered electrons from ${ }^{4} \mathrm{He}$, as observed along the dispersive detector axis. Insets show the same spectra on a logarithmic scale. Vertical lines indicate the acceptance of the detectors. The hatched region indicates the estimated quasielastic spectrum. The shaded region shows the contribution from the target aluminum endcaps.

thickness of the cryogenic cell walls. The asymmetry of this fraction, predominantly quasielastic scattering, was determined from [3]

$$
A_{\mathrm{QE}}^{\mathrm{PV}}=\frac{N_{p} \sigma_{p} A_{p}^{\mathrm{PV}}+N_{n} \sigma_{n} A_{n}^{\mathrm{PV}}}{N_{p} \sigma_{p}+N_{n} \sigma_{n}},
$$

which is $\sim-2 \mathrm{ppm}$ at these kinematics. A conservative uncertainty of $100 \%$ was used for this quantity.

\section{$3.2{ }^{4} \mathrm{He}$ quasielastic}

The optical properties of the spectrometer act to transform momentum differences at the target to spatial differences at the focal plane. For scattering from ${ }^{4} \mathrm{He}$, there are no excited states between the elastic peak and the nuclearbreakup threshold, which lies $19 \mathrm{MeV} / c$ away. Careful positioning of the elastic peak over the HAPPEX detector, in the focal plane, is crucial to minimizing the background from the quasielastic processes.

Special runs were taken to determine the detector location and orientation in the spectrometer focal plane. This is accomplished by setting the spectrometer to fully illuminate the focal plane with the fairly isotropic distribution of inelastic scattered electrons from solid targets. Measurements made from the ${ }^{4} \mathrm{He}$ target were then made at varying spectrometer settings to optimize the elastic peak over the HAPPEX detector.

The rise of the quasielastic spectrum is then observed, in relation to the detector edge in the focal plane (shown in fig. 3). This rise is estimated with a cut on the difference in the reconstructed momentum and the calculated scattered momentum that would be expected from elastic scattering. All inelastic events lie beyond a $19 \mathrm{MeV}$ threshold in this cut; events below this threshold are shown, hatched, in fig. 3 .
To account for reconstruction resolution, an upper limit of quasielastic contamination was established cutting on this momentum difference at $15 \mathrm{MeV}$, resulting in a fraction from quasielastic ${ }^{4} \mathrm{He}$ to be $(0.15 \pm 0.15) \%$ of the total rate observed in the detector. The asymmetry from this background is calculated to be $(-1.5 \pm 10.0) \mathrm{ppm}$ from eq. (4).

\subsection{Rescatter}

Some fraction of the detector signal arises from electrons that are rescattered inside of the spectrometer. This fraction is based on the following integral:

$$
f_{\mathrm{rs}}=\int_{0}^{E_{\text {elastic }}} \mathrm{d} E^{\prime} P_{\mathrm{rs}}\left(E^{\prime}\right) R\left(E^{\prime}\right)
$$

where $E^{\prime}$ is the energy of the scattered electron, $P_{\mathrm{rs}}$ is the ADC weighted probability of an electron with this energy to rescatter in the spectrometer and produce a signal in the detector, and $R\left(E^{\prime}\right)$ is the ratio of the inelastic crosssection to the elastic cross-section:

$$
R\left(E^{\prime}\right)=\frac{\left(\frac{\mathrm{d} \sigma}{\mathrm{d} \Omega \mathrm{d} E^{\prime}}\right)_{\text {inelastic }}}{\left(\frac{\mathrm{d} \sigma}{\mathrm{d} \Omega}\right)_{\text {elastic }}}
$$

Measurement of $P_{\mathrm{rs}}\left(E^{\prime}\right)$ is performed by increasing the spectrometer dipole field to force the elastic trajectories to trace those taken by the lower-energy inelastics at the production momentum setting. $R\left(E^{\prime}\right)$ is then measured by decreasing the dipole field. The resulting product between these two functions is integrated from the $E^{\prime}=0$ to the elastic energy. From this model, the background fraction from rescattering within the spectrometer is calculated to be $f_{\mathrm{rs}}=(0.25 \pm 0.15) \%$ for the helium run, and less than $0.10 \%$ for the hydrogen run. The asymmetry for these small fractions accounts for the estimated mix of inelastic and elastic radiative tail, at $(-1.5 \pm 3.0) \mathrm{ppm}$ for helium and $(-4 \pm 4)$ ppm for hydrogen.

Studies of the source of rescattered detector signals were also used to estimate an upper limit on the fraction from exposed iron of $\sim 10^{-5}$. With a maximum asymmetry of $\sim 200 \mathrm{ppm}$, this source of background contributed to a negligible uncertainty in each measurement.

\section{Conclusion}

An analysis of the $Q^{2}$ and backgrounds for the recent HAPPEX measurements has yielded precise results that keep the total systematic error in the parity-violating asymmetry less than the statistical precision (4\% relative error for helium and $0.1 \mathrm{ppm}$ for hydrogen). The techniques used provide a useful standard for future parity violation experiments endeavoring to obtain smaller relative measurements. 


\section{References}

1. J. Alcorn et al., Nucl. Instrum. Methods Phys. Res. A 522, 294 (2004).
2. N. Liyanage, Optics calibration of the Hall A high resolution spectrometers using the new $C++$ optimizer, JLab-TN-02$012,2002$.

3. M.J. Musolf, T.W. Donnelly, Nucl. Phys. A 549, 509 (1992). 